\title{
Is there still some reason for classic presentation of Amyotrophic Lateral Sclerosis described in the literature?
}

\begin{abstract}
Marco Orsini ${ }^{1 *}$, Acary Souza Bulle Olveira ${ }^{2}$, Carlos Henrique Melo Reis ${ }^{3}$, Jacqueline Fernandes do Nascimento ${ }^{3}$, Nicolle Nunes ${ }^{3}$, Antônio Marcos da Silva Catharino ${ }^{3}$, Valéria Camargo Silveira ${ }^{3}$, Adriana Leico Oda ${ }^{2}$, Mauricio Santanna Júnior ${ }^{4}$ and Marcos RG de Freitas ${ }^{5}$
\end{abstract}

${ }^{1}$ University of Iguaçu and University of Vassouras, Brazil

${ }^{2}$ Federal University of São Paulo, Brazil

${ }^{3}$ Iguaçu University and Hospital Geral da Posse, Brazil

${ }^{4}$ Federal University of Rio de Janeiro, Brazil

*Corresponding author: Marco Orsini, University of Iguaçu and Master's Program in Neurology - University of Vassouras; Iguassu University - UNIG, Brazil.

Email: orsinimarco@hotmail.com
Received Date: June 19, 2021

Published Date: July 09, 2021

\section{Opinion}

Obviously, a lot has changed regarding Amyotrophic Lateral Sclerosis (ALS). That old and exemplary definition made by Chacort, of a neurological disease, of a progressive, degenerative and inexorable character, with "isolated" depletion of the upper and lower motor neurons no longer seems so limited to words. ALS is already considered a systemic disease; not only because of new findings that circumvent motor manifestations, but because of genetic codes that signal a form of evolution of hereditary cases. Speaking of that 5-8 year survival after the onset of the first signs and symptoms, the increase in new technologies, associated with a different way of medicating and treating, changed the natural history of book chapters; even with the cruelty with which it strips and compromises patients in various topics related to their functional abilities. We are looking for a picture of Amyotrophic Lateral Sclerosis described in detail in the literature [1-2].

Charcot3, one of the best neurologists in the world, should "return" with his knowledge and unique clinical dissection of his cases, and to her the right to redescribe this new spectrum of presentation of ALS. We focus on what is known about ALS and where the research is going - from the small steps of extending longevity, improving therapies, conducting clinical trials and compiling population registries, to the global goals of establishing measures that protect against onset and finding the triggers for this neurodegenerative disorder [4]. In cases of hereditary ALS, much has become history with the advancement of molecular biology. In patients with ALS/FTD (Fronto-Temporal Dementia), for example, the neuroinflammation mechanism characterized by innate immune responses of tissue-resident glial cells is uniformly present in end-stage pathology. Human imaging studies and rodent models support that neuroinflammation begins at early stages of disease pathogenesis. In addition, changes in circulating immune cell populations and cytokines are found in ALS/FTD patients, and there is evidence of an auto-inflammatory state (humoral system) [5]. Increased levels of pro-inflammatory IL-6, IL-8, and nitrite and significantly decreased endogenous antioxidant GSH levels could identify these humoral constituents as systemic biomarkers for ALS. However, systemic changes in IL-2, IL-5, and IL-6 levels determined between visits in ALS patients might indicate adaptive immune system responses dependent on current disease stage [6]. ALS has broad genetic and hypothesized environmental causes and phenotypic variability. And what about sporadic ALS cases? These are even more unpredictable. In clinical practice, diagnostic difficulties mostly arise with patients who present either with only upper motor neuron, or with only lower motor neuron signs. In addition, patients with ALS may also have other nonmotor findings, such as changes in sensitivity, cognition problems, dysautonomia. It may be difficult to distinguish ALS with clinically 
predominant lower motor neuron involvement from alternative diagnoses including spinal atrophies of adult onset, Kennedy's disease, inclusion body myositis and motor neuropathies with conduction blocks 4-[7]. Although the degeneration predominantly affects the motor system, cognitive and behavioural symptoms have been described for over a century. In ALS, it is not possible to define a standard, nor to dare to scrutinize the survival of these patients. We have a certain medical rationality coupled with experience and dedication to the study of this merciless disease; but we still know little about its mysterious pathophysiological framework. We take advantage of and dedicate this opinion article to Professor Marcos RG de Freitas, neurologist, student and eternal apprentice of Professor Antonio Rodrigues de Mello.

\section{Acknowledgement}

None.

\section{Conflict of Interest}

No conflict of interest.

\section{References}

1. Belsh JM (1999) Diagnostic challenges in ALS. Neurology 53(8 Suppl 5): S26-30; discussion S35-6.

2. Phukan J, Pender NP, Hardiman O (2007) Cognitive impairment in amyotrophic lateral sclerosis. Lancet Neurol 6(11): 994-1003.

3. Katz JS, Dimachkie MM, Barohn RJ (2015) Amyotrophic Lateral Sclerosis: A Historical Perspective. Neurol Clin 33(4): 727-34.

4. Kiernan MC, Vucic S, Cheah BC, Turner MR, Eisen A, et al. (2011) Amyotrophic lateral sclerosis. Lancet 377(9769): 942-55.

5. McCauley ME, Baloh RH (2019) Inflammation in ALS/FTD pathogenesis. Acta Neuropathol 137(5): 715-730.

6. Zesiewicz TA, Jahan I, Shytle RD, Kim SH, Sanberg CD, et al. (2015) Humoral factors in ALS patients during disease progression. J Neuroinflammation 12: 127.

7. Orsini M, Oliveira AB, Nascimento OJ, Reis CH, Leite MA, et al. (2015) Amyotrophic Lateral Sclerosis: New Perpectives and Update. Neurol Int 7(2): 5885. 